\title{
Matrix-type certified reference materials for quality control of metal determination from solid environmental and vegetation samples
}

\section{ANDA GABRIELA TENEA ${ }^{1,2}$, GABRIELA GEANINA VASILE*1 ${ }^{1}$, GINA ALINA CATRINA ${ }^{1}$, MIHAELA MURESEANU ${ }^{2}$}

\author{
${ }^{I}$ National Research and Development Institute for Industrial Ecology - ECOIND, 71-73 Drumul Podu Dambovitei Str., \\ 060652, Bucharest, Romania,*gabriela.vasile@incdecoind.ro \\ ${ }^{2}$ Craiova University, Science Faculty, Chemistry Department, 107i Bucharest Road, Craiova, Romania
}

\begin{abstract}
In the context of monitoring environmental factors, metals are one of the major analytical components. Applying appropriate determination methods and obtaining accurate results is a requirement imposed on environmental laboratories that perform quality control of water, soil, waste or vegetation. This study presents some examples of certified reference materials for quality control of the results of toxic metal determination from solid environmental and vegetation samples. The analyzed and verified metals were $\mathrm{As}, \mathrm{Cd}, \mathrm{Cr}, \mathrm{Cu}, \mathrm{Ni}, \mathrm{Pb}$ and $\mathrm{Zn}$. The pre-treatment of the samples, the determination methods of metals and the obtained results are also presented. Inductively Coupled Plasma Optical Emission Spectrometry (ICP-EOS) and Inductively Coupled Plasma Mass Spectrometry (ICP-MS) techniques are suitable for low metal concentrations, while ICP-EOS and Flame Atomic Absorption Spectrometry (FAAS) methods can be used at high concentrations.
\end{abstract}

Keywords: toxic metals, ICP-EOS, Flame AAS, CRMs, environmental matrices

\section{INTRODUCTION}

Due to the advanced industrialization during the last decades of the past century and the first of this century, many areas became contaminated with toxic metals, considerable quantities being found both in soil, sediments, groundwater and surface water.

The monitoring and rehabilitation studies have as a starting point the investigation of the quality of environmental factors affected by anthropogenic activities, such as: mining, metallurgical industry, machine building industry, intensive agriculture, etc. Even if some mines have been closed or abandoned, the surrounding areas remain a potential danger to fauna, flora, the aquatic environment and human health [1-4].

Different types of waste with varied metal content are stored either in specially designed landfills, which over time can produce discharges of toxic substances into the environment, or directly on the ground as long as the content of toxic substances is below certain limits imposed by laws $[5,6]$.
The high metal content in contaminated soils, sediments, waste, biological sludge must be accurately determined using appropriate analytical techniques, so the composition of the complex matrices must not interfere with the determination [7].

In this context, in several international projects, matrix-type Certified Reference Materials (CRMs) have been developed, containing certified values of toxic metal content and the associated uncertainty.

Several institutions provide such complex matrices for soils (organic rich soil, sandy soil, road dust, industrial soil), sediments (estuarine, coastal, lake or river sediment), waste (fly ash, sewage sludge amended soil), plant tissue (hay powder, clover, lichen, tomato leaves, apple leaves, plankton, rice, etc.) or animal organs (tuna muscle, cod liver, etc.). Among these, we can mention the best known, namely: European Commission Joint Research Center - JRC (800 CRMs of the BCR- and IRMM-brands as well as the ERM-branded materials that were produced by the JRC) or US Department of Commerce - 
National Institute of Standards and Technology NIST. Obtaining such materials implies the application of well-established rules, which ensure the homogeneity and stability of the samples, as well as the assigned value and the uncertainty for each element or compound [8].
The international requirements imposed by the EN ISO 17025/2018 standard enforce accredited laboratories around the world to verify their analytical results for the determined pollutants [9]. Thus, the use of matrix-type certified reference materials has become a common practice in such laboratories (Table 1).

Table 1. Examples of CRMs in environmental analysis

\begin{tabular}{lll}
\hline \multicolumn{1}{c}{ CRMs } & \multicolumn{1}{c}{ Matrix type } & \multicolumn{1}{c}{ Aqua regia extractable content } \\
\hline ERM-CC 141 & Loam Soil & $\mathrm{As}, \mathrm{Cd}, \mathrm{Co}, \mathrm{Cr}, \mathrm{Cu}, \mathrm{Hg}, \mathrm{Mn}, \mathrm{Ni}, \mathrm{Pb}, \mathrm{Zn}$ \\
ERM-CC018 & Sandy Soil & $\mathrm{As}, \mathrm{Cd}, \mathrm{Cr}, \mathrm{Co}, \mathrm{Cu}, \mathrm{Pb}, \mathrm{Hg}, \mathrm{Ni}, \mathrm{V}, \mathrm{Zn}$ \\
RTC SPE-001 & Soil & $\mathrm{Al}, \mathrm{As}, \mathrm{Ba}, \mathrm{B}, \mathrm{Cd}, \mathrm{Ca}, \mathrm{Cr}, \mathrm{Co}, \mathrm{Cu}, \mathrm{Fe}, \mathrm{Li}, \mathrm{Pb}, \mathrm{Mg}$, \\
WQB-1 & Sediment & $\mathrm{Mn}, \mathrm{Hg}, \mathrm{Mo}, \mathrm{Ni}, \mathrm{K}, \mathrm{Se}, \mathrm{Ag}, \mathrm{Sb}, \mathrm{Na}, \mathrm{Sr}, \mathrm{Sn}, \mathrm{Ti}, \mathrm{V}, \mathrm{Zn}$ \\
RTC CRM016 & Sediment & $\mathrm{Al}, \mathrm{As}, \mathrm{Co}, \mathrm{Cu}, \mathrm{Pb}, \mathrm{Fe}, \mathrm{Mn}, \mathrm{Hg}, \mathrm{Ni}, \mathrm{Se}, \mathrm{V}, \mathrm{Zn}$ \\
& Sewage sludge from & $\mathrm{Mn}, \mathrm{Hg}, \mathrm{Mo}, \mathrm{Ni}, \mathrm{K}, \mathrm{Se}, \mathrm{Ag}, \mathrm{Sb}, \mathrm{Na}, \mathrm{Sr}, \mathrm{Sn}, \mathrm{Ti}, \mathrm{V}, \mathrm{Zn}$ \\
BCR 146R & industrial origin & $\mathrm{Cd}, \mathrm{Co}, \mathrm{Cr}, \mathrm{Cu}, \mathrm{Hg}, \mathrm{Mn}, \mathrm{Ni}, \mathrm{Pb}, \mathrm{Zn}$ \\
NIST 2782 & Industrial sludge & $\mathrm{As}, \mathrm{Cd}, \mathrm{Cr}, \mathrm{Cu}, \mathrm{Pb}, \mathrm{Hg}, \mathrm{Mo}, \mathrm{Ni}, \mathrm{Se}, \mathrm{Zn}$ \\
CRM 029 & Sewage sludge & $\mathrm{Al}, \mathrm{As}, \mathrm{Ba}, \mathrm{B}, \mathrm{Cd}, \mathrm{Ca}, \mathrm{Cr}, \mathrm{Co}, \mathrm{Cu}, \mathrm{Fe}, \mathrm{Pb}, \mathrm{Mg}, \mathrm{Mn}$, \\
BCR 483 & Sewage sludge amended soil $\mathrm{Cd}, \mathrm{Cr}, \mathrm{Cu}, \mathrm{Ni}, \mathrm{Pb}, \mathrm{Zn}$ \\
BCR 176R & Fly ash & $\mathrm{As}, \mathrm{Cd}, \mathrm{Co}, \mathrm{Cr}, \mathrm{Cu}, \mathrm{Fe}, \mathrm{Ni}, \mathrm{Pb}, \mathrm{Sb}, \mathrm{Se}, \mathrm{Tl}, \mathrm{Zn}$ \\
ERM-CE464 & Tuna fish & $\mathrm{Hg} \mathrm{total} \mathrm{CH}$ Hg \\
BCR 482 & Lichen & $\mathrm{Al}, \mathrm{As}, \mathrm{Cd}, \mathrm{Cr}, \mathrm{Cu}, \mathrm{Hg}, \mathrm{Ni}, \mathrm{Pb}, \mathrm{Zn}$ \\
NIST 1515 & Apple leaves & $\mathrm{Al}, \mathrm{B}, \mathrm{Ba}, \mathrm{Cd}, \mathrm{Ca}, \mathrm{Cu}, \mathrm{Fe}, \mathrm{Pb}, \mathrm{Mg}, \mathrm{Mn}, \mathrm{Hg}, \mathrm{Mo}, \mathrm{Ni}$, \\
& & $\mathrm{K}, \mathrm{Na}, \mathrm{V}, \mathrm{Zn}$ \\
NIST 1573a & Tomato leaves & $\mathrm{Al}, \mathrm{Sb}, \mathrm{As}, \mathrm{Ba}, \mathrm{B}, \mathrm{Cd}, \mathrm{Ca}, \mathrm{Cr}, \mathrm{Cu}, \mathrm{Co}, \mathrm{Fe}, \mathrm{Mn}, \mathrm{Hg}$, \\
\end{tabular}

Another way of testing the obtained results is by participating in proficiency testing (PT) schemes, where the entire applied procedure is tested (pretreatment, determination, reporting results). There are specialized international organizations that organize annually such schemes, these being diversified according to the pollutants and the matrix type (water, soil, waste, sediment, plant tissue). A database for such PTs in which the topics, the type of matrice

\section{EXPERIMENTAL PART}

\section{Methods}

In order to determine the metal content in solid environmental samples (soil, sediment, fly ash, sewage sludge), FAAS and ICP-EOS techniques were applied.

Table 2 presents the operational parameters for the method that uses ICP-EOS technique [11], while the performance parameters (quantification limit, accuracy and measurement and the accreditation of the organization that organizes the scheme are presented is EPTIS database [10].

The aim of this study is to present the results obtained by using various CRMs to check the metal content ( $\mathrm{As}, \mathrm{Cd}, \mathrm{Cu}, \mathrm{Cr}, \mathrm{Ni}, \mathrm{Pb}, \mathrm{Zn}$ ) from different environmental matrices (soil, sediment, fly ash, sewage sludge and vegetable tissues) as well as the applied analytical techniques for metal determination.

uncertainty) are listed in table 3 . The working conditions [12] and performance parameters for FAAS method are presented in table 4.

In the case of vegetation samples, due to their low metal contents, ICP-MS and ICP-EOS methods were applied. The operational parameters, respectively the performance 
parameters of the applied ICP-MS method are presented in tables 5 and 6 .

Table 2. Operating parameters for ICP-EOS AVIO 500 equipment

\begin{tabular}{|c|c|c|c|}
\hline \multirow{2}{*}{\multicolumn{2}{|c|}{ Spectrometer parameters }} & \multicolumn{2}{|c|}{\begin{tabular}{|l} 
Plasma parameters \\
\end{tabular}} \\
\hline & & Argon flow rate $15 \mathrm{~L} / \mathrm{min}$ & RF power $1400 \mathrm{~W}$ \\
\hline $\begin{array}{l}\text { Purge gas flow } \\
\text { rate: normal } \\
\text { Replicates: } 3 \text { times }\end{array}$ & $\begin{array}{l}\text { Delay time } \\
40 \text { s }\end{array}$ & $\begin{array}{l}\text { Auxiliary agent flow rate: } \\
0.2 \mathrm{~L} / \mathrm{min} \\
\text { Nebulizer flow rate: } \\
0.7 \mathrm{~L} / \mathrm{min}\end{array}$ & $\begin{array}{l}\text { Plasma view: } \\
\text { Axial } \\
\text { View distance: } \\
15.0 \mathrm{~mm} \\
\end{array}$ \\
\hline \multicolumn{4}{|c|}{ Processing spectral peaks } \\
\hline $\begin{array}{l}\text { Peak Algorithm: } \\
\text { Points per peak: }\end{array}$ & $\begin{array}{l}\mathrm{x} \text { area } \\
\text { oints }\end{array}$ & \multicolumn{2}{|c|}{ Spectral corrections: background correction } \\
\hline
\end{tabular}

Table 3. Performance parameters of the methods applied with the ICP-EOS AVIO 500 equipment

\begin{tabular}{|c|c|c|c|c|}
\hline Element & $\begin{array}{l}\text { Wavelength, } \\
\text { nm / matrix }\end{array}$ & $\begin{array}{l}\text { LOQ, } \\
\mathrm{mg} / \mathrm{kg}\end{array}$ & $\begin{array}{c}\text { Precision, } \\
\%\end{array}$ & $\begin{array}{c}\text { Uncertainty, } \\
\%\end{array}$ \\
\hline As & $\begin{array}{c}188.979 \text { (vegetation) } \\
197.197 \text { (solid samples) }\end{array}$ & 0.75 & 4.7 & 14. \\
\hline Cd & $\begin{array}{c}228.802 \text { (vegetation) } \\
214.440 \text { (solid sample) }\end{array}$ & 0.08 & 3.8 & 0.9 \\
\hline $\mathrm{Cr}$ & 267.716 & 0.04 & 2.4 & 13.3 \\
\hline $\mathrm{Cu}$ & $\begin{array}{c}324,754 \text { (vegetation) } \\
327.393 \text { (solid samples) }\end{array}$ & 0.05 & 5.3 & 11.7 \\
\hline $\mathbf{N i}$ & $\begin{array}{c}231.604 \text { (vegetation) } \\
341.476 \text { (solid sample) }\end{array}$ & 0.11 & 4.8 & 11.7 \\
\hline $\mathbf{P b}$ & $\begin{array}{c}220.353 \text { (vegetation) } \\
217.000 \text { (solid sample) }\end{array}$ & 0.33 & 2.7 & 9.4 \\
\hline $\mathbf{Z n}$ & 206.200 & 0.11 & 3.2 & 10.0 \\
\hline
\end{tabular}

Table 4. FAAS - Performance parameters and working conditions

\begin{tabular}{ccccccc}
\hline Element & $\begin{array}{c}\text { Wavelength, } \\
\text { nm }\end{array}$ & Flame type & $\begin{array}{c}\text { Background } \\
\text { correction }\end{array}$ & $\begin{array}{c}\text { LOQ, } \\
\mathbf{m g} / \mathbf{k g}\end{array}$ & $\begin{array}{c}\text { Precision, } \\
\mathbf{\%}\end{array}$ & $\begin{array}{c}\text { Uncertainty, } \\
\mathbf{\%}\end{array}$ \\
\hline $\mathrm{Cd}$ & 228.8 & air / acetilene & deuterium & 2.1 & 5.56 & 9.8 \\
\hline $\mathrm{Cu}$ & 324.8 & air / acetilene & deuterium & 6.0 & 3.82 & 15.0 \\
\hline $\mathrm{Ni}$ & 232.0 & air / acetilene & deuterium & 14.0 & 4.61 & 9.4 \\
\hline $\mathrm{Pb}$ & 217.0 & air / acetilene & deuterium & 16.0 & 5.21 & 9.7 \\
\hline $\mathrm{Zn}$ & 213.9 & air / acetilene & deuterium & 3.0 & 2.83 & 12.8 \\
\hline
\end{tabular}

Table 5. Operating parameters for ICP-MS spectrometer

\begin{tabular}{|c|c|}
\hline $\begin{array}{l}\text { Delay time: } 60 \mathrm{~s} \\
\text { Replicates: } 3 \text { times }\end{array}$ & $\begin{array}{l}\text { Purge gas flow: normal } \\
\text { Peristaltic pump: } 1.5 \mathrm{~mL} / \mathrm{min}\end{array}$ \\
\hline \multicolumn{2}{|c|}{$\begin{array}{l}\text { Tune parameters } \\
\text { Plasma parameters }\end{array}$} \\
\hline Plasma flow rate: $15 \mathrm{~L} / \mathrm{min}$ & RF Power: $1550 \mathrm{~W}$ \\
\hline Auxiliary flow rate: $0.90 \mathrm{~L} / \mathrm{min}$ & Plasma view: axial \\
\hline Nebulizer Pump: 0.10rps & RF matching: $1.30 \mathrm{~V}$ \\
\hline \multicolumn{2}{|c|}{ Plasma mode } \\
\hline Plasma Mode: General Purpose & Sample Depth: $10 \mathrm{~mm}$ \\
\hline \multicolumn{2}{|c|}{ Cell parameters } \\
\hline He Flow: $4.3 \mathrm{~mL} / \mathrm{min}$ & Octp Bias: $-8.0 \mathrm{~V}$ \\
\hline \multicolumn{2}{|c|}{ Spectral peak processing } \\
\hline Peak algorithm: Peak area & Peak pattern: 3 points \\
\hline Replicates: 3 times & Integration time: $0.2001 \mathrm{sec}$. \\
\hline
\end{tabular}


Table 6. Performance parameters for ICP-MS spectrometer

\begin{tabular}{cccccccc}
\hline Element & $\begin{array}{c}\text { LOQ, } \\
\mathbf{m g} / \mathbf{k g}\end{array}$ & $\begin{array}{c}\text { Precision, } \\
\mathbf{\%}\end{array}$ & $\begin{array}{c}\text { Uncertainty, } \\
\mathbf{\%}\end{array}$ & Element & $\begin{array}{c}\text { LOQ, } \\
\mathbf{m g} / \mathbf{k g}\end{array}$ & $\begin{array}{c}\text { Precision, } \\
\boldsymbol{\%}\end{array}$ & $\begin{array}{c}\text { Uncertainty, } \\
\mathbf{\%}\end{array}$ \\
\hline $\mathrm{As}$ & 0.15 & 2.14 & 11.6 & $\mathrm{Ni}$ & 0.21 & 1.20 & 13.5 \\
$\mathrm{Cd}$ & 0.19 & 1.92 & 15.4 & $\mathrm{~Pb}$ & 0.27 & 1.95 & 12.5 \\
$\mathrm{Cr}$ & 0.24 & 1.14 & 14.5 & $\mathrm{Zn}$ & 0.16 & 1.03 & 12.8 \\
$\mathrm{Cu}$ & 0.17 & 0.95 & 11.7 & & & & \\
\hline
\end{tabular}

All experimental tests were performed in an accredited laboratory according to EN ISO $17025 / 2018$ [9], respecting the requirements for

\section{Materials and equipment}

Multielements Certified Reference Material ME 21, $100 \mathrm{mg} / \mathrm{L}$ (Sigma-Aldrich)

Reference Material (RM) Quality Control Standard 21, $100 \mathrm{mg} / \mathrm{L}$ (LGC)

Hydrochloric acid $\geq 30 \%$ TraceSelect for trace analysis (Fluka)

Nitric acid ultra trace grade 69\% (Scharlau)

Hydrogen peroxide solution, trace select ultra, $\geq$ $30 \%$ for trace analysis (Sigma Aldrich)

\section{Samples preparation}

The content of metals extracted in aqua regia solution from the soil, sediment and sewage sludge samples, was determined. To $1 \mathrm{~g}$ of solid sample, $9 \mathrm{~mL} \mathrm{HCl}$ and $3 \mathrm{~mL} \mathrm{HNO}_{3}$ were added. For the pretreatment of these solid samples (soil, traceability and quality assurance of the analytical determinations results.

Inductively Coupled Plasma Optical Emission Spectrometer (ICP-OES) Perkin Elmer AVIO 500

Flame Atomic Absorption Spectrometer (FAAS) Thermo Scientific M6 Dual Inductively Coupled Plasma Mass Spectrometer (ICP-MS) 7900 Agilent Technologies Microwave Oven Ethos Up Milestone

sediment, and sewage sludge), a digestion program was applied (table 7). After cooling, the solutions were filtered and brought quantitatively to a $50 \mathrm{~mL}$ volumetric flask with ultrapure water.

Table 7. Microwave program for digestion of soil, sediment and sewage sludge samples

\begin{tabular}{lcccc}
\hline & Power & Time & $\begin{array}{c}\text { Temperature T1 } \\
\text { (inside vessels) }\end{array}$ & $\begin{array}{c}\text { Temperature T2 } \\
\text { (outside vessels) }\end{array}$ \\
\hline Stage 1 & $1800 \mathrm{~W}$ & 15 minutes & up to $180^{\circ} \mathrm{C}$ & $120^{\circ} \mathrm{C}$ \\
Stage 2 & $1800 \mathrm{~W}$ & 20 minutes & $180^{\circ} \mathrm{C}$ & $120^{\circ} \mathrm{C}$ \\
Stage 3 & - & 30 minutes & cooling & cooling \\
\hline
\end{tabular}

Vegetation samples $(0.5 \mathrm{~g})$ were pretreated with a mixture of $8 \mathrm{~mL} \mathrm{HNO}_{3}$ and $2 \mathrm{~mL} \mathrm{H}_{2} \mathrm{O}_{2}$ and then subjected to the digestion program presented in table 8 . The resulting solutions were filtered on low porosity filter paper and brought to the mark with ultrapure water in 25 $\mathrm{mL}$ volumetric flask.

Table 8. Microwave program for digestion of apple leave and lichen samples

\begin{tabular}{lccc}
\hline & Power & Time & Temperature \\
\hline Stage 1 & $1800 \mathrm{~W}$ & 15 minutes & up to $200^{\circ} \mathrm{C}$ \\
Stage 2 & $1800 \mathrm{~W}$ & 15 minutes & $200^{\circ} \mathrm{C}$ \\
Stage 3 & - & 30 minutes & cooling \\
\hline
\end{tabular}

For each matrix type and each applied digestion program, blank samples were used in order to verify the interference of reagents used during sample preparation.

The metals from the following CRMs: loam soil (ERM-CC 141), sandy soil (ERM-CC 018), sediment (WQB-1), fly ash (176 R), industrial 
sludge (NIST 2782) were extracted in aqua regia mixture. The analyzed vegetation CRMs were: apple leaves (NIST 1515), lichen (BCR 482).
Each experiment was performed in duplicate, the reported values being the average values.

\section{RESULTS AND DISCUSSION}

The results obtained for the analyzed metals (As, $\mathrm{Cd}, \mathrm{Cr}, \mathrm{Cu}, \mathrm{Ni}, \mathrm{Pb}, \mathrm{Zn}$ ) in the CRMs are presented in this section. Thus, the obtained data respectively FAAS results) are compared to the certified values from the quality certificates of the used CRMs (tables $9 \div 13$ ).

for solid environmental samples (ICP-EOS,

Table 9. Loam soil ERM-CC 141(JRC, IRMM brand), mg/kg dry matter

\begin{tabular}{cccc}
\hline \multirow{2}{*}{ Element } & \multirow{2}{*}{ Certified value } & \multicolumn{2}{c}{ Determined values } \\
\cline { 3 - 4 } & & ICP-EOS & FAAS \\
\hline $\mathrm{As}$ & $7.5 \pm 1.4$ & $8.8 \pm 1.3$ & - \\
$\mathrm{Cr}$ & $31 \pm 4$ & $30.4 \pm 4.0$ & - \\
$\mathrm{Cu}$ & $12.4 \pm 0.9$ & $11.6 \pm 1.4$ & $11.5 \pm 1.7$ \\
$\mathrm{Ni}$ & $21.9 \pm 1.6$ & $20.3 \pm 2.4$ & $22.1 \pm 2.1$ \\
$\mathrm{~Pb}$ & $32.2 \pm 1.4$ & $30.9 \pm 2.9$ & - \\
\hline
\end{tabular}

Table 10. Sandy soil ERM-CC 018, mg/kg dry matter

\begin{tabular}{cccc}
\hline \multirow{2}{*}{ Element } & \multirow{2}{*}{ Certified value } & \multicolumn{2}{c}{ Determined values } \\
\cline { 3 - 4 } & & ICP-EOS & FAAS \\
\hline $\mathrm{As}$ & $22.9 \pm 1.3$ & $21.7 \pm 3.1$ & - \\
$\mathrm{Cd}$ & $5.4 \pm 0.5$ & $5.9 \pm 0.5$ & - \\
$\mathrm{Ni}$ & $25.8 \pm 1.8$ & $27.3 \pm 3.2$ & $24.4 \pm 2.3$ \\
$\mathrm{~Pb}$ & $289 \pm 10$ & $298 \pm 28$ & $281 \pm 27$ \\
\hline
\end{tabular}

Table 11. Sediment WQB-1, mg/kg dry matter

\begin{tabular}{cccc}
\hline \multirow{2}{*}{ Element } & \multirow{2}{*}{ Certified value } & \multicolumn{2}{c}{ Determined values } \\
\cline { 3 - 4 } & & ICP-EOS & FAAS \\
\hline $\mathrm{As}$ & $23 \pm 1.8$ & $24.7 \pm 3.5$ & - \\
$\mathrm{Cu}$ & $79.6 \pm 16.1$ & $66.4 \pm 7.8$ & $63.5 \pm 9.5$ \\
$\mathrm{Ni}$ & $61.5 \pm 17.6$ & $57.1 \pm 6.7$ & $59.7 \pm 5.6$ \\
\hline
\end{tabular}

Table 12. Fly Ash $176 \mathrm{R}, \mathrm{mg} / \mathrm{kg}$ dry matter

\begin{tabular}{cccc}
\hline \multirow{2}{*}{ Element } & \multirow{2}{*}{ Certified value } & \multicolumn{2}{c}{ Determined values } \\
\cline { 3 - 4 } & & ICP-EOS & FAAS \\
\hline $\mathrm{Cu}$ & $1000 \pm 70$ & $1013 \pm 119$ & $989 \pm 148$ \\
$\mathrm{~Pb}$ & $5000 \pm 500$ & $4572 \pm 430$ & $4563 \pm 443$ \\
$\mathrm{Zn}$ & $16800 \pm 400$ & $17039 \pm 1700$ & $16670 \pm 2130$ \\
\hline
\end{tabular}

Table 13. Industrial sludge NIST 2782, mg/kg dry matter

\begin{tabular}{cccc}
\hline \multirow{2}{*}{ Element } & \multirow{2}{*}{ Certified value } & \multicolumn{2}{c}{ Determined values } \\
\cline { 3 - 4 } & & ICP-EOS & FAAS \\
\hline $\mathrm{Cu}$ & $2594 \pm 52$ & $2626 \pm 307$ & $2555 \pm 383$ \\
$\mathrm{~Pb}$ & $574 \pm 11$ & $569 \pm 53$ & $573 \pm 56$ \\
$\mathrm{Zn}$ & $1254 \pm 196$ & $1062 \pm 106$ & $1170 \pm 150$ \\
\hline
\end{tabular}

The reported values for all metals in all solid matrices that were analysed are within the confidence intervals according to the quality certificates data. 
Tables 14 and 15 show the results obtained for ICP-EOS, respectively ICP-MS techniques. vegetation samples after metal determination by

Table 14. Apple Leaves NIST $1515, \mathrm{mg} / \mathrm{kg}$ dry matter

\begin{tabular}{cccc}
\hline \multirow{2}{*}{ Element } & \multirow{2}{*}{ Certified value } & \multicolumn{2}{c}{ Determined values } \\
\cline { 3 - 4 } & & ICP-EOS & ICP-MS \\
\hline $\mathrm{Cd}$ & $0.0132 \pm 0.0015$ & $<0.08$ & $0.013 \pm 0.0013$ \\
$\mathrm{Cu}$ & $5.69 \pm 0.13$ & $5.58 \pm 0.88$ & $5.57 \pm 0.56$ \\
$\mathrm{Ni}$ & $0.936 \pm 0.094$ & $1.025 \pm 0.132$ & $1.02 \pm 0.10$ \\
$\mathrm{~Pb}$ & $0.470 \pm 0.024$ & $<1.5$ & $0.49 \pm 0.05$ \\
$\mathrm{Zn}$ & $12.45 \pm 0.43$ & $12.75 \pm 1.67$ & $12.18 \pm 1.22$ \\
\hline
\end{tabular}

Table 15. Lichen BCR 482, $\mathrm{mg} / \mathrm{kg}$ dry matter

\begin{tabular}{cccc}
\hline \multirow{2}{*}{ Element } & \multirow{2}{*}{ Certified value } & \multicolumn{2}{c}{ Determined values } \\
\cline { 3 - 4 } & & ICP-EOS & ICP-MS \\
\hline $\mathrm{As}$ & $0.85 \pm 0.07$ & - & $0.91 \pm 0.09$ \\
$\mathrm{Cd}$ & $0.56 \pm 0.02$ & - & $0.57 \pm 0.06$ \\
$\mathrm{Cr}$ & $4.12 \pm 0.15$ & $5.58 \pm 0.88$ & $5.57 \pm 0.56$ \\
$\mathrm{Cu}$ & $7.03 \pm 0.19$ & $6.87 \pm 0.80$ & $7.17 \pm 0.72$ \\
$\mathrm{Ni}$ & $2.47 \pm 0.07$ & $2.53 \pm 0.30$ & $2.44 \pm 0.24$ \\
$\mathrm{~Pb}$ & $40.9 \pm 1.4$ & $41.3 \pm 3.9$ & $40.5 \pm 4.1$ \\
$\mathrm{Zn}$ & $100.6 \pm 2.2$ & $99.6 \pm 10.0$ & $98.7 \pm 9.9$ \\
\hline
\end{tabular}

Table 16 contains the results obtained after The table also presents the $\mathrm{Z}$ scores obtained for participating in an inter-laboratory comparison the reported data, all values falling within the -2 scheme for a soil sample analysis, the obtained $\div 2$ accepted range of standard deviation. values being compared to the assigned values.

Table 16. LGC sample 14/2019, results of PTS, mg/kg dry matter

\begin{tabular}{cccccc}
\hline \multirow{2}{*}{ Element } & \multirow{2}{*}{ Assigned value } & \multicolumn{2}{c}{ ICP-EOS } & \multicolumn{2}{c}{ FAAS } \\
\cline { 3 - 5 } & & Value & Z score & Value & Z score \\
\hline $\mathrm{As}$ & $32.9 \pm 4.2$ & $27.9 \pm 4.2$ & -1.44 & - & - \\
$\mathrm{Cd}$ & $6.28 \pm 0.63$ & $5.14 \pm 0.51$ & -1.72 & - & - \\
$\mathrm{Cr}$ & $153 \pm 15.3$ & $145.4 \pm 17.4$ & -0.47 & $145.0 \pm 18.7$ & -0.49 \\
$\mathrm{Cu}$ & $112.8 \pm 16.8$ & $106.3 \pm 16.8$ & -0.57 & $118.3 \pm 13.6$ & -0.47 \\
$\mathrm{Ni}$ & $43.9 \pm 4.4$ & $37.8 \pm 5.3$ & -1.39 & $45.7 \pm 4.4$ & 0.44 \\
$\mathrm{~Pb}$ & $226.7 \pm 22.7$ & $228.3 \pm 31.5$ & 0.07 & $226.7 \pm 22.6$ & 0.00 \\
$\mathrm{Zn}$ & $815 \pm 81.5$ & $898.2 \pm 90.2$ & 1.02 & $935 \pm 87$ & 1.47 \\
\hline
\end{tabular}

\section{CONCLUSIONS}

The results obtained in the verification of some methods applied for the determination of toxic metals both from solid environmental (soil, sediment, ash waste, respectively sewage sludge) and vegetation samples indicated that the applied ICP-EOS, FAAS, respectively ICPMS techniques led to good results both on CRM matrices and in the case of participation in a proficiency test scheme.

\section{ACKNOWLEDGEMENTS}

The authors acknowledge the financial support offered by The National Research Program "Nucleu" through contract no 20N/2019, Project code PN 19040101. 


\section{REFERENCES}

[1] DINU, C., VASILE, G.G., BULEANDRA, Monit. Assess., 190, no. 10, 2018, 609, M., POPA, D.E., GHEORGHE, S., https://doi.org/10.1007/s10661-018-6983-x

UNGUREANU E.M., J. Soil Sediment., 20, no. [8] OLIVARES, I.R.B., SOUZA, G.B., 4, 2020, p. 2141. NOGUIRA, A.R.A., TOLEDO, G.T.K.,

[2] DINU, C., UNGUREANU, E.M., VASILE, MARCKI, D.C., Trends. Anal. Chem., 100, G.G., KIM, L., SIMION, M., IONESCU, I., 2018, p. ENE, C., Rev. Chim., 69, no. 1, 2018, p. 14 https://doi.org/10.1016/j.trac.2017.12.013

[3] KIM L., VASILE G.G., STANESCU B., [9] EN ISO/IEC 17025:2018 - General DINU C., ENE C., Rev. Chim., 67, no. 8, 2016, requirements for the competence of testing and p. 1441

[4] GUTIERREZ, M., QIU, X., ZACHARY, calibration laboratories.

J.C., ZACHARY, T.L., Minerals, 10, no. 3, https://www.eptis.bam.de/eptis/WebSearch/main 2020, 247, [30.06.2020].

https://doi.org/10.3390/min10030247.

[11] EN 16170/2017 - Sludge, Treated Biowaste

[5] GHEORGHE S., VASILE G.G., STOICA

C., NITA-LAZAR M., LUCACIU I., BANCIU

A., Rev. Chim., 67, no. 8, 2016, p. 1469.

[6] KHAN, M.A., KHAN, S., KHAN, A., ALAM, M., Sci. Total Environ., 601-602, 2017, p. https://doi.org/10.1016/j.scitotenv.2017.06.030.

[7] VASILE, G.G., POPA, D.E., And Soil - Determination of elements using Inductively Coupled Plasma Optical Emission Spectrometry (ICP-OES).

[12] ISO 11047/1998 - Soil quality Determination of cadmium, chromium, cobalt, copper, lead, manganese, nickel and zinc BULEANDRA, M., DAVID, I.G., Environ. spectrometric methods. 